\title{
INITIAL BOUNDARY VALUE PROBLEMS FOR NONCONVEX HYPERBOLIC CONSERVATION LAWS WITH RELAXATION*
}

\author{
LING $\mathrm{HSIAO}^{\dagger}$ AND HAILIANG $\mathrm{LI}^{\ddagger}$
}

\begin{abstract}
The initial boundary value problem (IBVP) for the $2 \times 2$ nonconvex conservation laws with relaxation proposed by Jin and Xin [11] is considered in the present paper under the subcharacteristic condition. The global existence of smooth solutions for this IBVP are proved, and the large time behavior is analyzed. These tend time-asymptotically to either a travelling wave, or a superposition of two travelling waves respectively, corresponding to different kinds of boundary effects.
\end{abstract}

1. Introduction. In this paper, we investigate the global existence and asymptotic behavior of solutions for the $2 \times 2$ conservation laws with relaxation in $\Omega=$ $\{(x, t): x \geq \sigma t, t \geq 0\}$

$$
\left\{\begin{array}{l}
u_{t}+v_{x}=0 \\
v_{t}+a u_{x}=-\frac{1}{\varepsilon}(v-f(u)),
\end{array}\right.
$$

with initial and boundary values given by

$$
\left\{\begin{array}{l}
(u, v)(x, 0)=\left(u_{0}, v_{0}\right)(x),\left(u_{0}, v_{0}\right)(+\infty)=\left(u_{+}, v_{+}\right), x \geq 0 \\
\left.u(x, t)\right|_{x=\sigma t}=u_{-}, \quad t \geq 0
\end{array}\right.
$$

or

$$
\left\{\begin{array}{l}
(u, v)(x, 0)=\left(u_{0}, v_{0}\right)(x),\left(u_{0}, v_{0}\right)(+\infty)=\left(u_{+}, v_{+}\right), x \geq 0, \\
\left.v(x, t)\right|_{x=\sigma t}=v_{-}, \quad t \geq 0,
\end{array}\right.
$$

where $v_{ \pm}=f\left(u_{ \pm}\right)$with $f(u)$ nonconvex, and $\sigma^{2}<a$ is required to make sure the well-posedness of the problem.

Equation (1.1) is a simple form to the general conservation laws with relaxation proposed by Jin and Xin [11], where the systems were used to numerically approximate a set of corresponding hyperbolic conservation laws with non-oscillation, which is exactly a local relaxation approximation. Equation (1.1) is also a simplified form to the general $2 \times 2$ conservation laws with relaxation proposed by T.-P. Liu [20]

The effect of relaxation is often taken into consideration when the physical situation of an investigated material is in nonequilibrium. This phenomenon is generally modelled as hyperbolic conservation laws with source terms. There are many different kinds of relaxation phenomena which arise in nature, such as gases in thermo-nonequilibrium states, kinetic theory of mono-atomic gases, water waves, viscoelasticity with memory, chromatography, and so on [5], [36].

As the relaxation time $\varepsilon$ goes to $0^{+}$, we formally obtain from (1.1) the following scalar conservation laws

$$
u_{t}+f(u)_{x}=0
$$

${ }^{*}$ Received Jan. 11, 1999; revised Feb. 10, 2000.

†Institute of Mathematics, Academia Sinica, Beijing 100080, P. R. China (hsiaol@sun.iehp.ac.cn). Supported by the Natural National Science Fundation of China.

$\ddagger$ Institute of Mathematics, Academia Sinica, Beijing 100080, P. R. China and SISSA, Via Beirut 2-4, 34014 Trieste, Italy (lihl@sissa.it). 
The relation between $2 \times 2$ conservation laws with relaxation and their corresponding equilibrium equation was shown theoretically by T.-P. Liu [20] first. Therein, he justified the nonlinear stability criteria for elementary waves and showed the stability of them. He also gave the subcharacteristic condition which governs the propagation of disturbances over strong wave forms. For (1.1), the corresponding subcharacteristic condition is

$$
-\sqrt{a}<f^{\prime}(u)<\sqrt{a}
$$

which, from now on, is assumed true for (1.1).

We consider the initial boundary value problem (IBVP) (1.1) and (1.2) or (1.3) in the present paper. We are interested in how the boundary influences the global existence of smooth solutions to this IBVP and their large time behavior. It is proved by Nishibata [31] that the appearance of boundary makes it impossible for a travelling wave to be an exact solution to this IBVP. We want to know whether the small perturbations of weak elementary waves are still time-asymptotically stable in the presence of boundary. That means if there are still some waves or time-asymptotic states, such as travelling waves, expansion wave, or their superposition and so on, to which the global smooth solutions (if they exist ) of (1.1) and (1.2) or (1.3) tend time-asymptotically. Boundaries influence a real physical situation by causing the appearance of boundary layer. This important physical phenomena [34] occurs, for instance, in describing the interaction of fluid molecules with the molecules of the solid boundary, and has been modelled with the scalar viscous conservation laws by Xin [37] and with the Boltzmann equation by J.-G. Liu and Xin $[18,19]$. It is shown (see case $\mathcal{B}$ below) that the given boundary value and the initial informations at infinity sometimes can not determine the expected time-asymptotic state for the IBVP of (1.1), which is different from the Cauchy problem for (1.1) where the time-asymptotic state can be uniquely determined by the initial informations at infinity. Therefore, a criteria is required to determine the expected time-asymptotic states for the IBVP (1.1) and (1.2) or (1.3). In this paper, the criteria is that the "boundary layer", the difference of boundary value and the asymptotic state restricted at boundary, can be controllable in $L^{1}$ norm.

The aim of this paper is to find whether the small perturbation of travelling wave for (1.1) is still stable time-asymptotically in the presence of moving boundary. For simplicity, we assume $\varepsilon=1$. It was shown in [16] that under the Oleinik entropy condition, i.e.,

$$
f(u)-f\left(u_{ \pm}\right)-s\left(u-u_{ \pm}\right)\left\{\begin{array}{l}
<0, \text { if } u_{+}<u<u_{-} \\
>0, \text { if } u_{+}>u>u_{-}
\end{array}\right.
$$

and $\mathrm{R}-\mathrm{H}$ condition, i.e.,

$$
-s\left(u_{+}-u_{-}\right)+\left(f\left(u_{+}\right)-f\left(u_{-}\right)\right)=0,
$$

there exits a unique travelling wave $\left(\dot{U}_{s}, V_{s}\right)(\eta)(\eta=x-s t)$ for (1.1) up to a shift, which satisfies $\left(U_{s}, V_{s}\right)( \pm \infty)=\left(u_{ \pm}, v_{ \pm}\right)$with $v_{ \pm}=f\left(u_{ \pm}\right)$.

In addition, since we consider the influence of moving boundary to the relaxation system, we have to compare the moving speeds of the boundary and the travelling wave in order to find an expected time-asymptotic state. We discuss two cases: $s>\sigma$ and $s<\sigma$.

Case $\mathcal{A}: s>\sigma$. For simplicity, we only consider the case $s>0>\sigma$ and only deal with the IBVP (1.1) and (1.2). Since the boundary layer $\left(u_{-}-U_{s}((\sigma-s) t)\right)$ 


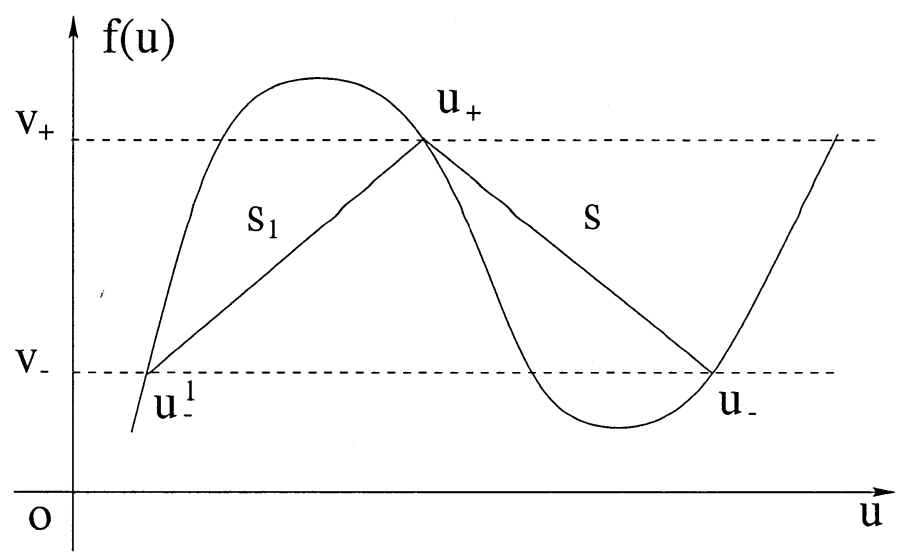

FIG. 1.1.

belongs to $L^{1}\left(R_{+}\right)$, we may expect $\left(U_{s}, V_{s}\right)$ to be the time-asymptotic state of the global solution (if it exists) to (1.1) and (1.2).

It is well known that for scalar conservation laws with viscosity and $2 \times 2$ conservation laws with relaxation, a small perturbation of a travelling wave at most gives rise to a translation. But it is more complicated in our case because of the effects of moving boundary. In fact, similarly to that used by T.-P. Liu and Yu [22], Matsumura and Mei [26], and Mei and Rubino [28], a shift function $x(t), t \geq 0$, which satisfies an ordinary differential equation coupled with an unknown term $\left(v(\sigma t, t)-v_{-}\right)$, is used to overcome the difficulties caused by boundary. Moreover, we have to use higher order weighted energy estimates to overcome the nonconvexity of $f$ and to control the term $u_{x}(\sigma t, t)$ caused by the moving boundary. The main result shows that if the initial data is a small perturbation of the travelling wave $\left(U_{s}, V_{s}\right)\left(x-s t+x_{0}\right)$ restricted on $\Omega$ with $t=0$, then there exists a global solution of (1.1)-(1.2) which tends to $\left(U_{s}, V_{s}\right)(x-s t+x(t))$ as $t \rightarrow+\infty$ where $x(0)=x_{0}<0$ and $x(+\infty)=x_{\infty}<+\infty$, provided that $\left|x_{0}\right|$ is big enough.

Case $\mathcal{B}: s<\sigma$. The boundary layer is not controllable if we still expect $\left(U_{s}, V_{s}\right)$ to be the asymptotic state. Therefore, an appropriate time-asymptotic state is expected. In fact, for the IBVP (1.1) and (1.2) the expected time-asymptotic state, due to the influence of boundary, can be only a part of another travelling wave $\left(U_{\sigma}, V_{\sigma}\right)(x-\sigma t)$ of $(1.1)$ which satisfies that $\left(U_{\sigma}, V_{\sigma}\right)(+\infty)=\left(u_{+}, v_{+}\right), U_{\sigma}^{\prime}(\eta)<0$, $\eta \in R$ and $U_{\sigma}(0)=u_{-}$. This is similar to the phenomena observed by Nisibata [31] and Mei and Rubino [28]. Furthermore, to control the boundary terms, we assume that $\sigma \geq 0$ and $\sigma>f^{\prime}\left(u_{-}\right)$. The result for the IBVP (1.1)-(1.2) illustrates that a small perturbation of the travelling wave $\left(U_{\sigma}, V_{\sigma}\right)$ restricted on $\Omega$ with $t=0$ admits the global existence of solution to (1.1)-(1.2) which tends to $\left(U_{\sigma}, V_{\sigma}\right)(x-\sigma t)$ as $t \rightarrow+\infty$.

While for the IBVP (1.1) and (1.3), there appears new interesting phenomena. For simplicity, we only consider the case $\sigma=0$. The expected time-asymptotic state here is $\left(U_{p}, V_{p}\right)(x, t)$, a superposition of $\left(U_{s}, V_{s}\right)(x-s t)$ and $\left(U_{s_{1}}, V_{s_{1}}\right)\left(x-s_{1} t\right)$ with $s_{1}>\sigma>s$, which can be obtained as follows. We assume that the nonconvex $f$ satisfies the following property, which can be illustrated by figure 1.1

Property 1. There exist $u_{-}^{i}\left(i=1,2\right.$ for simplicity) with $f\left(u_{-}^{i}\right)=v_{-}$and $u_{-}^{2}=u_{-}$, such that for $s_{1}>0>s_{2}=s$, the Oleinik's entropy condition and Rankine- 


\section{Hugniot condition hold}

$$
\begin{aligned}
& s_{1}\left(u_{-}^{1}-u_{+}\right)=f\left(u_{-}^{1}\right)-f\left(u_{+}\right), h_{1}(u)>0, \text { for } u_{-}^{1}<u<u_{+} \\
& s_{2}\left(u_{-}-u_{+}\right)=f\left(u_{-}\right)-f\left(u_{+}\right), h_{2}(u)<0, \text { for } u_{+}<u<u_{-} .
\end{aligned}
$$

where $h_{i}(u)=f(u)-f\left(u_{+}\right)-s_{i}\left(u-u_{+}\right), \quad i=1,2 .$.

This means that the interaction of the shock $\left(u_{-}, u_{+}, s<0\right)$ for (1.4) with the boundary forms a new shock $\left(u_{-}^{1}, u_{+}, s_{1}>0\right)$. Thus, the asymptotic state for (1.1) and (1.3) is expected to be

$$
\left(U_{p}, V_{p}\right)=\left(U_{s_{1}}, V_{s_{1}}\right)+\left(U_{s}, V_{s}\right)-\left(u_{+}, v_{+}\right)
$$

We will show that if the initial data is a small perturbation of an appropriate selected $\left(U_{p}, V_{p}\right)$ then the IBVP (1.1) and (1.3) owns a global solution which tends to the $\left(U_{p}, V_{p}\right)$ as $t \rightarrow+\infty$. This phenomena is quite different from that for the IBVP (1.1)(1.2) and from the stability of travelling wave for (1.1) observed by Liu, Wang, and Yang [16]. But it is somehow similar to the phenomena observed by Hsiao and Pan [7] where the nonlinear stability of two-mode shock profiles is proved. Such phenomena is new for the initial boundary value problems of conservation laws with relaxation and is also an answer to the unsolved problems proposed in [28], where the IBVP for (1.1) with a different boundary condition from (1.2) or (1.3) is discussed, which will be introduced afterwards.

Recently, there appear a number of results on $2 \times 2$ conservation laws with relaxation. For the general model, the stability of travelling waves with decay rates for the Cauchy problem and the stability theory but without decay rate for the initial boundary problem were studied by Zingano [39], Nishibata [31], and Nishibata and Yu [33] respectively. The problem on the convergence to the diffusion waves was shown by Chern [4], Yao and Zhu [38]. Related results on the relaxation limit were established by Chen and T.-P. Liu [3], Chen, Levermore and T.-P. Liu [2], Natalini [30], and Marcati and Rubino [24].

For the simplest model (1.1), the stability of travelling wave solutions for the Cauchy problem was discussed by H. L. Liu, Woo and Yang [17], H. L. Liu, Wang and Yang [16], Mascia and Natalini [25], and finally Mei and Yang [29]. The convergence to the travelling wave solutions, as the relaxation time goes to zero, was recently considered by Jin and H. L. Liu [10]. The $L^{1}$ relaxation limit of (1.1) with boundary effect in the BV-framework was discussed by Wang and Xin [35]. Furthermore, the numerical computation and the properties of entropy solution for the model (1.1) were given by Jin and Levermore [9], and Jin [8]. For Jin-Xin's model in higher space dimensions, the stability of travelling waves was shown by Luo and Xin [23].

Regarding the stability of travelling waves to the IBVP for other model equations of hyperbolic conservation laws, we refer to those interesting works in $[14,21,22,26]$.

This paper is arranged as follows. After some preliminaries about the travelling wave are introduced in section 2 , we will list the main results for (1.1) about case $\mathcal{A}$ and case $\mathcal{B}$ with different initial and boundary conditions in section 3 . These results will be proved successively in section 4 .

Notation $L^{2}$ denotes the space of square integrable functions on $R$ or $R_{+}$with the norm

$$
\|f\|=\left(\int|f(x)|^{2} d x\right)^{1 / 2}
$$


$H^{l}(l \geq 0)$ denotes the Sobolev space of $f$ with $\partial_{x}^{j} f, j=0,1, \cdots, l$, belonging to $L^{2}$ and with the norm

$$
\|f\|_{l}=\left(\sum_{j=0}^{l}\left\|\partial_{x}^{j} f\right\|^{2}\right)^{1 / 2} .
$$

For a given weight function $K_{j}(x)=\left(1+x^{2}\right)^{(\beta+j) / 2}, x \in R_{+}, j \in R, L_{\beta+j}$ represents the space of weighted square integrable functions with norm

$$
|f|_{\beta+j}=\left(\int_{0}^{+\infty} K_{j}(x)|f(x)|^{2} d x\right)^{1 / 2} .
$$

$H_{\beta}^{l}(l \geq 0)$ denotes the weighted Sobolev space of $f$ satisfying $\partial_{x}^{j} f \in L_{\beta+j}(j=$ $0,1,2, \cdots, l)$ and normed by

$$
\|f\|_{H_{\beta}^{l}}=\left(\int_{0}^{+\infty} \sum_{j=0}^{l}\left|\partial_{x}^{j} f\right|_{\beta+j}^{2} d x\right)^{1 / 2} .
$$

For a given function $K_{j}(x, t)=\left(1+(x+d(t)-s t)^{2}\right)^{(\beta+j) / 2}$ where $s$ is a constant and $d(t)$ a smooth function, $C^{0}\left(0, T ; H_{\beta}^{l}\right)$ or $L^{2}\left(0, T ; H_{\beta}^{l}\right)$ will denote the space of functions $f(x, t)$ satisfying the condition that $t:[0, T] \rightarrow H_{\beta}^{l}$ is continuous or square integrable respectively. The norms will respectively be given by

$$
\max _{t \in[0, T]}\|f(\cdot, t)\|_{H_{\beta}^{l}}, \quad \sqrt{\int_{0}^{t}\|f(\cdot, \tau)\|_{H_{\beta}^{l}} d \tau} .
$$

2. Preliminaries. A travelling wave solution to system (1.1) is a solution $\left(U_{s}, V_{s}\right)(\eta),(\eta=x-s t)$, satisfying $(1.1)$ and $\left(U_{s}, V_{s}\right)( \pm \infty)=\left(u_{ \pm}, v_{ \pm}\right)$with $v_{ \pm}=$ $f\left(u_{ \pm}\right)$, namely,

$$
\left\{\begin{array}{l}
-s U_{s}^{\prime}+V_{s}^{\prime}=0 \\
-s V_{s}^{\prime}+a U_{s}^{\prime}=f(U)-V \\
\left(U_{s}, V_{s}\right)( \pm \infty)=\left(u_{ \pm}, v_{ \pm}\right)
\end{array}\right.
$$

Here $^{\prime}=d / d \eta$.

Integrating the first equation of $(2.1)$ over $(-\infty, \eta]$ and $[\eta,+\infty)$ respectively, and noticing $\left(U_{s}, V_{s}\right)( \pm \infty)=\left(u_{ \pm}, v_{ \pm}\right)$, we have

$$
-s U_{s}+V_{s}=-s u_{ \pm}+v_{ \pm}=-s u_{ \pm}+f\left(u_{ \pm}\right),
$$

which shows that, the speed $s$ and the two states $\left(u_{ \pm}, v_{ \pm}\right)$satisfy the RankineHugoniot condition

$$
s=\frac{f\left(u_{+}\right)-f\left(u_{-}\right)}{u_{+}-u_{-}} .
$$

Substituting (2.2) into the second equation of (2.1) we obtain

$$
\left(a-s^{2}\right) U_{s}^{\prime}=f\left(U_{s}\right)-f\left(u_{ \pm}\right)-s\left(U_{s}-u_{ \pm}\right) \equiv: h\left(U_{s}\right) .
$$


It is well-known that the ordinary differential equation (2.4) has a smooth solution if and only if the Rankine-Hugoniot condition (2.3) and the Oleinik's entropy condition

$$
h(u)=f(u)-f\left(u_{ \pm}\right)-s\left(u-u_{ \pm}\right) \begin{cases}<0, & u_{+}<u<u_{-} \\ >0, & u_{+}>u>u_{-}\end{cases}
$$

holds. This entropy condition implies

$$
f^{\prime}\left(u_{+}\right)<s<f^{\prime}\left(u_{-}\right)
$$

or

$$
f^{\prime}\left(u_{+}\right)=s<f^{\prime}\left(u_{-}\right) \quad \text { or } \quad f^{\prime}\left(u_{+}\right)<s=f^{\prime}\left(u_{-}\right) \quad \text { or } \quad f^{\prime}\left(u_{ \pm}\right)=s .
$$

Condition (2.6) is the well-known Laxian shock condition. Here we will call it the nondegenerate shock condition and we will refer to each one of the possibility in (2.7) as the degenerate shock condition, or the contact shock condition.

As shown in Figure 1.1, there are two waves to (1.1) connecting different state points. One is the back wave connecting $\left(u_{ \pm}, v_{ \pm}\right)$, another is the forward wave connecting $\left(u_{+} / u_{-}^{1}, v_{ \pm}\right)$. Now we firstly state some preliminaries on the back wave to (1.1) connecting $\left(u_{ \pm}, v_{ \pm}\right)$, which, given by (1.2), satisfies (2.3), (2.5), $u_{+}<u_{-}$and $f\left(u_{+}\right)>f\left(u_{-}\right)$.

Lemma 2.1. [16] Assume $f \in C^{2}$, and the conditions (2.3) and (2.5) hold. There exists a unique solution $\left(U_{s}, V_{s}\right)(\eta)(\eta=x-s t)$ up to a shift to (1.1) with $0<s^{2}<a$ $(s<0)$, which satisfies

$$
\left(a-s^{2}\right) U_{s}^{\prime}=h\left(U_{s}\right)<0, \quad \text { for } \quad u_{+}<U_{s}<u_{-} .
$$

In addition, as $\eta \rightarrow \pm \infty$, it holds that for $f^{\prime}\left(u_{ \pm}\right) \neq s$

$$
\left|h\left(U_{s}\right)\right| \sim\left|\left(U_{s}-u_{ \pm}, V_{s}-v_{ \pm}\right)\right| \sim \exp \left\{-c_{ \pm}|\eta|\right\}
$$

and that for $f(u)=s u+\left(u-u_{ \pm}\right)^{(n+1)}, n=1,2,3 \cdots$,

$$
\left|h\left(U_{s}\right)\right|^{1 /(1+n)} \sim\left|\left(U_{s}-u_{ \pm}, V_{s}-v_{ \pm}\right)\right| \sim|\eta|^{-1 / n},
$$

where $c_{ \pm}=\left|f^{\prime}\left(u_{ \pm}\right)-s\right| /\left(a-s^{2}\right)>0$.

By Lemma 2.1, we have

Lemma 2.2. Assume $f \in C^{2}$. There exists a unique solution $\left(U_{\sigma}, V_{\sigma}\right)(\eta)(\eta=$ $x-\sigma t)$ to $(1.1)$ satisfying $\left(U_{\sigma}, V_{\sigma}\right)(+\infty)=\left(u_{+}, v_{+}\right), U_{\sigma}(0)=u_{-}$, and

$$
\left(a-\sigma^{2}\right) U_{\sigma}^{\prime}=h\left(U_{\sigma}\right)<0, \text { for } u_{+}<U_{\sigma}<u_{-} .
$$

In addition, as $\eta \rightarrow+\infty$, it holds that for $f^{\prime}\left(u_{+}\right) \neq \sigma$

$$
\left|h\left(U_{\sigma}\right)\right| \sim C\left|\left(U_{\sigma}-u_{+}, V_{\sigma}-v_{+}\right)\right| \rightarrow C \exp \left\{-\tilde{c}_{+}|\eta|\right\}
$$

and that for $f(u)=\sigma u+\left(u-u_{+}\right)^{(n+1)}, n=1,2,3, \cdots$,

$$
\left|h\left(U_{\sigma}\right)\right|^{1 /(1+n)} \sim C\left|\left(U_{\sigma}-u_{+}, V_{\sigma}-v_{+}\right)\right| \rightarrow C|\eta|^{-1 / n},
$$

where $\tilde{c}_{+}=\left|f^{\prime}\left(u_{+}\right)-\sigma\right| /\left(a-\sigma^{2}\right)>0$. 
Proof. We consider $f$ as a part of $\tilde{f} \in C^{2}$, which satisfies

$$
\begin{aligned}
& \tilde{f}=f, \text { for } u_{+} \leq u \leq u_{-}, \\
& \tilde{f}(u)-\sigma u<\tilde{f}\left(u_{+}\right)-\sigma u_{+}, \text {for } u_{+}<u<\tilde{u}_{-},
\end{aligned}
$$

where $\tilde{u}_{-}$is determined by $\tilde{f}\left(\tilde{u}_{-}\right)-\sigma \tilde{u}_{-}=\tilde{f}\left(u_{+}\right)-\sigma u_{+}$. Then Lemma 2.2 follows in terms of Lemma 2.1 .

We have also some results on the forward travelling wave solution to (1.1), which connects the states $\left(u_{+} / u_{-}^{1}, v_{ \pm}\right)$, satisfying the Rankine-Hugoniot condition (2.8) and Oleinik's entropy condition (2.9), i.e.,

$$
\begin{gathered}
s_{1}=\frac{v_{+}-v_{-}}{u_{+}-u_{-}^{1}}=\frac{f\left(u_{+}\right)-f\left(u_{-}^{1}\right)}{u_{+}-u_{-}^{1}}>0, \\
h_{1}(u)=f(u)-f\left(u_{+} / u_{-}^{1}\right)-s_{1}\left(u-u_{+} / u_{-}^{1}\right)>0, \quad u_{+}>u>u_{-}^{1},
\end{gathered}
$$

where $u_{-}^{1}<u_{+}, v_{-}=f\left(u_{-}^{1}\right)<f\left(u_{+}\right)=v_{+}$. The symbol " $u_{+} / u_{-}^{1}$ " express " $u_{+}$or $u_{-}^{1} "$. We consider first that the forward wave $\left(U_{s_{1}}, V_{s_{1}}\right)\left(x-s_{1} t\right)$ is nondegenerate, namely, the Laxian entropy condition holds

$$
f^{\prime}\left(u_{+}\right)<s_{1}<f^{\prime}\left(u_{-}^{1}\right) .
$$

Lemma 2.3. Assume $f \in C^{2}$, and the conditions (2.8), (2.9) and (2.10) hold. There exists a unique solution $\left(U_{s_{1}}, V_{s_{1}}\right)(\eta)\left(\eta=x-s_{1} t\right)$ up to a shift to (1.1) with $0<s_{1}^{2}<a\left(s_{1}>0\right)$, which satisfies $\left(U_{s_{1}}, V_{s_{1}}\right)( \pm \infty)=\left(u_{+} / u_{-}^{1}, v_{ \pm}\right)$and

$$
\left(a-s_{1}^{2}\right) U_{s_{1}}^{\prime}=h_{1}\left(U_{s_{1}}\right)>0, \quad \text { for } \quad u_{-}^{1}<U_{s_{1}}<u_{+} .
$$

In addition, as $\eta \rightarrow \pm \infty$, it holds that

$$
\left|h_{1}\left(U_{s_{1}}\right)\right| \sim\left|\left(U_{s_{1}}-u_{+} / u_{-}^{1}, V_{s_{1}}-v_{ \pm}\right)\right| \sim \exp \left\{-c_{ \pm}^{\prime}|\eta|\right\}
$$

where $c_{ \pm}^{\prime}=\left|f^{\prime}\left(u_{+} / u_{-}^{1}\right)-s_{1}\right| /\left(a-s_{1}^{2}\right)>0$.

Define the following weight function (see [27, 29]),

$$
Q\left(U_{s_{1}}\right)=\frac{\left(U_{s_{1}}-u_{-}^{1}\right)\left(u_{+}-U_{s_{1}}\right)}{h_{1}\left(U_{s_{1}}\right)}
$$

for $\left(U_{s_{1}}, V_{s_{1}}\right)$. There are properties for $Q$ given in $[27,29]$ as follows.

LEMMA 2.4. [27, 29] Let $\left(U_{s_{1}}, V_{s_{1}}\right)(\eta)$ be the travelling wave to (1.1) given by Lemma 2.3, then it holds, as $\eta \rightarrow \pm \infty$, that

$$
Q\left(U_{s_{1}}\right) \sim O(1), \quad\left(Q h_{1}\right)^{\prime \prime}\left(U_{s_{1}}\right)=-2, \quad\left|\frac{Q\left(U_{s_{1}}\right)_{\eta}}{Q\left(U_{s_{1}}\right)}\right|=O(1) \frac{u_{+}-u_{-}^{1}}{a-s_{1}^{2}}, i=1,2
$$


3. Main results. First, we consider the IBVP (1.1) and (1.2). For the case $0<-\sigma<s$, the difference between the boundary value and the travelling wave restricted at boundary $\left(u_{-}-U_{s}\left(x_{0}+(\sigma-s) t\right)\right)$ with a constant $x_{0}<0$ belongs to $L^{1}\left(R_{+}\right)$.

Set

$$
w_{1}(x)=-\int_{x}^{+\infty}\left(u_{0}(y)-U_{s}\left(y+x_{0}\right)\right) d y, z_{1}(x)=v_{0}(x)-V_{s}\left(x+x_{0}\right),
$$

and assume

$$
\int_{0}^{+\infty}\left(u_{0}(y)-U_{s}\left(y+x_{0}\right)\right) d y=0 .
$$

We only consider, for a technique reason, the case that $a$ is a big constant, $\mid u_{+}-$ $u_{-} \mid<<1$ and $f^{\prime}\left(u_{-}\right)>s>f^{\prime}\left(u_{+}\right)$with $s>\sqrt{a} / 2$. Then, the result for the IBVP (1.1)-(1.2) is

Theorem 3.1. Suppose that $f \in C^{5}$, conditions (2.3), (2.5), and (3.1) hold, and $\left(w_{1}, \partial_{x} w_{1}, z_{1}\right) \in\left(H_{\beta}^{3}\right)^{3}$ for $2 \beta>1$. Then if $\left(1+\left|x_{0}\right|^{\beta+3}\right)\left\|\left(w_{1}, \partial_{x} w_{1}, z_{1}\right)\right\|_{H_{\beta}^{3}}+\left|x_{0}\right|^{-1}<$ $\varepsilon_{1}$ for some $\varepsilon_{1}>0$, a global solution $(u, v)(x, t)$ to (1.1) and (1.2) exists and satisfies

$$
\sup _{\sigma t \leq x<+\infty}\left|(u, v)(x, t)-\left(U_{s}, V_{s}\right)(x-s t+x(t))\right| \rightarrow 0 \text {, as } t \rightarrow+\infty,
$$

where $x(t)$ satisfies the following ordinary differential equation

$$
\left\{\begin{aligned}
x^{\prime}(t)\left[u_{+}-U_{s}(\sigma t-s t+x(t))\right]+\sigma\left(u_{-}-U_{s}(\sigma t-s t+x(t))\right) & \\
& -\left(v(\sigma t, t)-V_{s}(\sigma t-s t+x(t))\right)=0 \\
x(0) & =x_{0}
\end{aligned}\right.
$$

and

$$
x(t) \in C^{1}(0,+\infty), \quad x^{\prime}(t) \in L^{1}(0,+\infty), \quad \text { and } x(+\infty)=x_{\infty}<+\infty .
$$

REMARK. (1) The value $x_{\infty}$ can be determined uniquely by

$$
\begin{aligned}
& \int_{0}^{+\infty}\left(u_{+}-U_{s}\left(x_{\infty}+\eta\right)\right) d \eta+\int_{-\infty}^{0}\left(u_{-}-U_{s}\left(x_{\infty}+\eta\right)\right) d \eta \\
& +\int_{0}^{+\infty}\left(u_{0}(x)-u_{+}\right) d x+\int_{0}^{+\infty}\left(v(\sigma t, t)-v_{-}\right) d t=0
\end{aligned}
$$

(2) Similar result is true for the IBVP (1.1) and (1.3).

For case $\sigma>s$, the difference between the boundary value and the travelling wave restricted at boundary $\left(u_{-}-U_{s}(d+(\sigma-s) t)\right)$ with $d$ a constant tends to $\left(u_{-}-u_{+}\right)$ as $t \rightarrow+\infty$. Therefore, we may expect $\left(U_{\sigma}, V_{\sigma}\right)(x-\sigma t)$ restricted on $\Omega$ as the timeasymptotic state.

Set

$$
w_{2}(x)=-\int_{x}^{+\infty}\left(u_{0}(y)-U_{\sigma}(y)\right) d y, \quad z_{2}(x)=v_{0}(x)-V_{\sigma}(x),
$$

we have the following theorem 
Theorem 3.2. Let $\sigma \geq 0,\left|u_{+}-u_{-}\right| \ll 1$ and $\sigma>f^{\prime}\left(u_{-}\right)$. Suppose that $f \in C^{2}$, condition (2.3) and (2.5) hold, $w_{2} \in H^{2}$, and $z_{2} \in H^{1}$. Then there exists a $\varepsilon_{2}>0$, such that if $\left\|w_{2}\right\|_{2}+\left\|z_{2}\right\|_{1}<\varepsilon_{2}$, a global solution $(u, v)(x, t)$ to (1.1) and (1.2) exists and satisfies

$$
\sup _{\sigma t \leq x<+\infty}\left|(u, v)(x, t)-\left(U_{\sigma}, V_{\sigma}\right)(x-\sigma t)\right| \rightarrow 0 \text {, as } t \rightarrow+\infty
$$

and

$$
\left|v(\sigma t, t)-V_{\sigma}(0)\right| \rightarrow 0 \text {, as } t \rightarrow+\infty \text {. }
$$

Then, we consider the IBVP (1.1) and (1.3). We only consider the case $s<$ $\sigma=0$, to which other cases corresponding to those for IBVP (1.1)-(1.2) are similar. The IBVP for (1.1) on quarterly plane was also discussed by Mei and Rubino [28]. Therein three kinds of boundary conditions

$$
v(0, t)=g(t)=\left\{\begin{array}{l}
v_{-}=f\left(u_{-}\right), \text {for } s>0 \\
v_{ \pm}=f\left(u_{ \pm}\right), \text {for } s=0 \\
v_{+}=f\left(u_{+}\right), \text {for } s<0
\end{array}\right.
$$

were considered. Where $s\left(u_{+}-u_{-}\right)=f\left(u_{+}\right)-f\left(u_{-}\right)$and the Oleinik's entropy condition holds. The time-asymptotic states $\left(U_{s}, V_{s}\right)(x, t)$ shown in [28] are either

$$
\left(U_{s}, V_{s}\right)(x, t)=\left(U_{s}, V_{s}\right)\left(x-s t+\alpha_{0}\right), \text { for } s \neq 0
$$

where $\alpha_{0}$ is a constant, or

$$
\left(U_{s}, V_{s}\right)(x, t)=\left(U_{s}(x+b(t)), v_{+}\right), \text {for } s=0,
$$

where $b(t)$ is a given function.

It is easy to verify that $\left(g(t)-V_{s}(0, t)\right) \in L^{1}\left(R_{+}\right)$in these cases. The authors [28] obtained the existence of global solutions and analyzed their asymptotic behaviors of them with time-decay rates. But for the cases when the boundary values are either

$$
g(t)=v_{-}=f\left(u_{-}\right), \text {for } s<0,
$$

or

$$
g(t)=v_{+}=f\left(u_{+}\right), \text {for } s>0,
$$

they have no results. In fact, $\left(g(t)-V_{s}\left(-s t+\alpha_{0}\right)\right)$ does not belong to $L^{1}\left(R_{+}\right)$in both cases (q.1)-(q.2). Therefore, different time-asymptotic states should be expected, which in fact is the superposition of the two travelling waves for the nonconvex $f$ satisfying the Property 1 shown in figure 1.1. For simplicity, we only consider the IBVP (1.1) and (1.3) for case (q.1) with nondegenerate waves, i.e.,

$$
f^{\prime}\left(u_{-}^{1}\right)>s_{1}>f^{\prime}\left(u_{+}\right), \quad f^{\prime}\left(u_{-}\right)>s>f^{\prime}\left(u_{+}\right) .
$$

The case (q.2) can be solved similarly.

For any given constants $x_{1}$ and $d_{0}$ satisfying $0<-d_{0}<x_{1}$, we make the essential assumption on the initial data $u_{0}$

$$
\int_{0}^{\infty}\left[u_{0}(x)-U_{s_{1}}\left(x+d_{0}\right)-U_{s}\left(x+x_{1}\right)+u_{+}\right] d x=0 .
$$


Denote

$$
\left(U_{p}, V_{p}\right)(x, t)=\left(U_{s_{1}}, V_{s_{1}}\right)\left(x-s_{1} t+d(t)+d_{0}\right)+\left(U_{s}, V_{s}\right)\left(x-s t+x_{1}\right)-\left(u_{+}, v_{+}\right),
$$

where $d(t)$ is the desired shift function chosen as the solution of the following ordinary differential equation

$$
\left\{\begin{aligned}
d^{\prime}(t) & {\left[u_{+}-U_{s_{1}}\left(d_{0}-s_{1} t+d(t)\right)\right] } \\
& =v_{-}-V_{s_{1}}\left(d_{0}-s_{1} t+d(t)\right)+v_{+}-V_{s}\left(x_{1}-s t\right) \\
d(0) & =0
\end{aligned}\right.
$$

It will be proved in Lemma 4.2 below that $d(t) \in C^{1}(0,+\infty), d^{\prime}(t) \in L^{1}(0,+\infty)$, and $d(t) \rightarrow d_{\infty}<+\infty$ as $t \rightarrow+\infty$, where the value of $d_{\infty}$ can be determined, by using the original idea of Matsumura and Mei to determine their shift [26], as

$$
d_{\infty}=\frac{1}{u_{+}-u_{-}^{1}}\left\{\int_{0}^{+\infty}\left(U_{s}\left(x+x_{1}\right)-u_{+}\right) d x+\int_{-\infty}^{0}\left(u_{-}^{1}-U_{s_{1}}\left(x+d_{0}\right)\right) d x\right\} .
$$

Set

$$
w_{0}(x)=-\int_{x}^{+\infty}\left(u_{0}(y)-U_{p}(y, 0)\right) d y, \quad z_{0}(x)=v_{0}(x)-V_{p}(x, 0) .
$$

We have

Theorem 3.3. Let $\sigma=0$. Suppose that $f \in C^{3}$, the conditions (ND), (3.2), (2.3), (2.5), (2.8) and (2.9) hold. Let $a>0$ be a big constant, $w_{0} \in H^{2}$ with $w_{0}(0)=0$, and $z_{0} \in H^{1}$. Then there exists a constant $\varepsilon_{0}>0$, such that if $\left\|w_{0}\right\|_{2}+\left\|z_{0}\right\|_{2}+\left|d_{0}\right|^{-1}+$ $x_{1}^{-1}<\varepsilon_{0}$, a global solution $(u, v)(x, t)$ to (1.1) and (1.3) exists uniquely and satisfies

$$
\sup _{x \in R_{+}}\left|(u, v)(x, t)-\left(U_{p}, V_{p}\right)(x, t)\right| \rightarrow 0, \text { as } t \rightarrow+\infty \text {. }
$$

REMARK. 1) The restriction of $a \gg 1$ means the requirement of a strong diffusion effect, which was used by H. Liu, Wang and Yang in [16], H. Liu, Woo and Yang in [17] and replaced with $\left|u_{+}-u_{-}\right| \ll 1$ by Mei and Yang [29] for the Cauchy problem. For the IBVP (1.1) and (1.3), due to the resolutions on boundary terms, Mei and Rubino [28] has to also use $a \gg 1$. But, in the present paper, it is not difficult to find in the late proof that for Theorems 3.3 the condition $a \gg 1$ can be replaced by that, $\left|u_{+}-u_{-}^{1}\right|$ is small enough such that

$$
a-\left(f^{\prime}\left(U_{p}\right)\right)^{2}-C_{b}\left|u_{+}-u_{-}^{1}\right|>0, \text { and } a-s_{1}^{2}-C_{b}\left|u_{+}-u_{-}^{1}\right|>0,
$$

with $C_{b}>0$ a constant.

2) The convergence rates of the solutions given by Theorem 3.3 are obtained in [6], where the corresponding cases for degenerate travelling waves are also discussed.

4. Reformulation of problems and proofs. The proofs of Theorem 3.1 and Theorem 3.2 can be completed by combining the methods used by T.-P. Liu and Nishihara [21], Matsumura and Mei [26], and Nishibata and Yu [33], we omit the detail here. We here only show Theorem 3.3. 
Let $\left(U_{s_{1}}, V_{s_{1}}\right)\left(x-s_{1} t\right)$ and $\left(U_{s}, V_{s}\right)(x-s t)$ be the forward and backward waves mentioned before. Let $(u, v)(x, t)$ be the solution to IBVP (1.1) and (1.3). Since $\left(U_{s}, V_{s}\right)\left(x-s t+x_{1}\right)$ satisfies $(2.1)$ and $\left(U_{s_{1}}, V_{s_{1}}\right)\left(x-s_{1} t+d(t)+d_{0}\right)$ satisfies

$$
\left\{\begin{array}{l}
\partial_{t} U_{s_{1}}-d^{\prime}(t) \partial_{x} U_{s_{1}}+\partial_{x} V_{s_{1}}=0 \\
\partial_{t} V_{s_{1}}-d^{\prime}(t) \partial_{x} V_{s_{1}}+a \partial_{x} U_{s_{1}}=f\left(U_{s_{1}}\right)-V_{s_{1}}
\end{array}\right.
$$

it follows, by (4.1), (2.1), and (3.3), that

$$
\left\{\begin{array}{l}
\partial_{t}\left(u-U_{p}\right)+d^{\prime}(t) \partial_{x} U_{s_{1}}+\partial_{x}\left(v-V_{p}\right)=0 \\
\partial_{t}\left(v-V_{p}\right)+d^{\prime}(t) \partial_{x} V_{s_{1}}+a \partial_{x}\left(u-U_{p}\right) \\
\quad=f(u)-f\left(U_{s_{1}}\right)-f\left(U_{s}\right)+f\left(u_{+}\right)-\left(v-V_{p}\right)
\end{array}\right.
$$

Integrating the first equation of $(4.2)$ over $[0,+\infty)$ and noticing that $v(0, t)=v_{-}$, we obtain

$$
\begin{aligned}
& \frac{d}{d t} \int_{0}^{+\infty}\left(u-U_{p}\right) d x+d^{\prime}(t)\left[u_{+}-U_{s_{1}}\left(-s_{1} t+d(t)+d_{0}\right)\right] \\
& \quad-\left(v_{-}-V_{s_{1}}\left(-s_{1} t+d(t)+d_{0}\right)+v_{+}-V_{s}\left(-s t+x_{1}\right)\right)=0 .
\end{aligned}
$$

It holds for $d(t)$ satisfying (3.4) that

$$
\frac{d}{d t} \int_{0}^{+\infty}\left(u-U_{p}\right)(x, t) d x=0, \quad t \geq 0
$$

Integrating it over $[0, t]$ and using (3.2) lead to

$$
\begin{aligned}
\int_{0}^{\infty}\left(u-U_{p}\right)(x, t) d x & =\int_{0}^{\infty}\left[u_{0}(x)-U_{p}(x, 0)\right] d x \\
& =\int_{0}^{\infty}\left[u_{0}(x)-U_{s_{1}}\left(x+d_{0}\right)-U_{s}\left(x+x_{1}\right)+u_{+}\right] d x \\
& =0 .
\end{aligned}
$$

Thus, we may define

$$
w(x, t)=-\int_{x}^{\infty}\left[u(y, t)-U_{p}(y, t)\right] d y, \quad z(x, t)=v(x, t)-V_{p}(x, t),
$$

to obtain

$$
\left\{\begin{array}{l}
\partial_{t} w+d^{\prime}(t)\left[U_{s_{1}}\left(x+\eta_{1}\right)-u_{+}\right]+z=0 \\
\partial_{t} z+d^{\prime}(t) \partial_{x} V_{s_{1}}\left(x+\eta_{1}\right)+a \partial_{x}^{2} w+z \\
\quad=f\left(U_{p}+w_{x}\right)-f\left(U_{s_{1}}\left(x+\eta_{1}\right)\right)-f\left(U_{s}\left(x+\eta_{2}\right)\right)+f\left(u_{+}\right)
\end{array}\right.
$$

where $\eta_{1}=:-s_{1} t+d(t)+d_{0}$ and $\eta_{2}=:-s t+x_{1}$.

Therefore, $w(x, t)$ satisfies the following equation

$$
w_{t t}+w_{t}-a w_{x x}+f^{\prime}\left(U_{p}\right) w_{x}=g_{1}(x, t)+g_{2}(x, t),
$$


where

$$
\left\{\begin{aligned}
g_{1}(x, t)= & d^{\prime}(t) V_{s_{1}}^{\prime}\left(x+\eta_{1}\right)+\left(s_{1}-d^{\prime}(t)\right) d^{\prime}(t) U_{s_{1}}^{\prime}\left(x+\eta_{1}\right) \\
& +\left(d^{\prime}(t)+d^{\prime \prime}(t)\right)\left[u_{+}-U_{s_{1}}\left(x+\eta_{1}\right)\right] \\
g_{2}(x, t)= & -\left\{f\left(U_{p}+w_{x}\right)-f\left(U_{s_{1}}\left(x+\eta_{1}\right)\right)\right. \\
& \left.-f\left(U_{s}\left(x+\eta_{2}\right)\right)-f^{\prime}\left(U_{p}\right) w_{x}+f\left(u_{+}\right)\right\}
\end{aligned}\right.
$$

with the initial and boundary values

$$
\left\{\begin{array}{l}
w(x, 0)=w_{0}(x), w_{t}(x, 0)=-z_{0}(x)+z_{0}(0) \frac{u_{+}-U_{s_{1}}\left(x+d_{0}\right)}{u_{+}-U_{s_{1}}\left(d_{0}\right)}=: w_{3}(x), x \geq 0 \\
w(0, t)=0, \quad t \geq 0
\end{array}\right.
$$

We reformulate Theorem 3.3 as follows.

THEOREM 4.1. Assume the hypotheses of Theorem 3.3 hold. Then the IBVP (4.7) and (4.9) has a unique global solution $w(x, t)$ satisfying

$$
\begin{aligned}
& \|w(\cdot, t)\|_{2}^{2}+\left\|w_{t}(\cdot, t)\right\|_{1}^{2}+\int_{0}^{t}\left[\left\|\sqrt{U_{s_{1}}^{\prime}} w(\cdot, \tau)\right\|^{2}+\left\|\left(w_{x}, w_{t}\right)(\cdot, \tau)\right\|_{1}^{2}\right] d \tau \\
\leq & C\left(\left\|w_{0}\right\|_{2}^{2}+\left\|z_{0}\right\|_{1}^{2}+e^{c_{-}^{\prime} d_{0} / 4}+e^{-c_{+} x_{1} / 4}\right) .
\end{aligned}
$$

Let $T>0$. Define the work spaces for (4.7) and (4.9) as

$$
X(0, T)=\left\{w \in C^{0}\left(0, T ; H^{2}\right) \cap L^{2}\left(0, T ; H^{2}\right), \quad w_{t} \in C^{0}\left(0, T ; H^{1}\right) \cap L^{2}\left(0, T ; H^{1}\right)\right\},
$$

and denote

$$
N(T)=\sup _{0 \leq t \leq T}\left\{\|w(\cdot, t)\|_{2}+\left\|w_{t}(\cdot, t)\right\|_{1}\right\}, \quad t \in[0, T] .
$$

The proof of Theorem 4.1 can be completed based on the following a priori estimates in Lemmas 4.2-4.4.

First, we have more accurate estimates on $d(t)$.

Lemma 4.2. Let $\left|d_{0}\right|^{-1}+x_{1}^{-1} \ll 1$. Then $d(t) \in C^{3}(0,+\infty), d^{\prime}(t) \in L^{1}(0,+\infty)$, and $|d(t)| \leq C$ for some constant, and

$$
d(t)-s_{1} t \leq-\frac{1}{2} s_{1} t, \quad t \in[0,+\infty)
$$

and

$$
\left|d^{\prime}(t)\right| \sim\left|d^{\prime \prime}(t)\right| \sim\left|d^{\prime \prime \prime}(t)\right| \sim\left\{e^{-c_{-}^{\prime}\left(s_{1} t-d_{0}\right) / 2}+e^{-c_{+}\left(|s| i+x_{1}\right) / 2}\right\}, t \in[0,+\infty)
$$

and

$$
d(t) \rightarrow d_{\infty}:=\frac{1}{u_{+}-u_{-}^{1}}\left\{\int_{0}^{\infty}\left[U_{s}\left(x+x_{1}\right)-u_{+}\right] d x+\int_{-\infty}^{0}\left[u_{-}^{1}-U_{s_{1}}\left(x+d_{0}\right)\right] d x\right\}
$$


as $t \rightarrow+\infty$.

Proof. By Lemma 2.3 and $d_{0}<0$, we get

$$
u_{+}-U_{s_{1}}\left(d_{0}\right)>0
$$

which means, in terms of Lemmas 2.1 and 2.3 and (3.4), that

$$
\left|d^{\prime}(0)\right|=\frac{1}{u_{+}-U_{s_{1}}\left(d_{0}\right)}\left|v_{-}-V_{s_{1}}\left(d_{0}\right)+v_{+}-V_{s}\left(x_{1}\right)\right| \leq \frac{1}{4} s_{1},
$$

provided that $\left|d_{0}\right|^{-1}+x_{1}^{-1} \ll 1$.

Therefore, it holds, for some $t_{0}>0$, that

$$
\left|d^{\prime}(t)\right| \leq \frac{1}{2} s_{1}, \quad 0 \leq t \leq t_{0}
$$

which yields, with $d(0)=0$, that

$$
|d(t)|=\left|\int_{0}^{t} d^{\prime}(\tau) d \tau\right| \leq \frac{1}{2} s_{1} t, \quad 0 \leq t \leq t_{0},
$$

and

$$
d(t)-s_{1} t \leq-\frac{1}{2} s_{1} t \leq 0, \quad 0 \leq t \leq t_{0}
$$

By Lemma 2.3 and (4.18), it follows

$$
u_{+}-U_{s_{1}}\left(-s_{1} t+d(t)+d_{0}\right) \geq u_{+}-U_{s_{1}}\left(d_{0}\right), \quad 0 \leq t \leq t_{0} .
$$

Then, by (3.4), (4.19) and Lemmas 2.1-2.3, we obtain

$$
\left|d^{\prime}(t)\right| \leq \frac{1}{4} s_{1}, \quad 0 \leq t \leq t_{0}
$$

Repeating above procedure, we can verify that (4.18) and (4.20) hold for all $t \in[0, \infty)$, namely, we have proved (4.11).

To prove (4.12), we first note that,

$$
\left|v_{-}-V_{s_{1}}\left(-s_{1} t+d(t)+d_{0}\right)\right| \leq C e^{-c_{-}^{\prime}\left(\frac{1}{2} s_{1} t+\left|d_{0}\right|\right)}
$$

and

$$
\left|u_{+}-U_{s_{1}}\left(-s_{1} t+d(t)+d_{0}\right)\right| \sim\left|u_{+}-u_{-}^{1}\right|
$$

which means, due to (4.11) and Eq. (3.4), that

$$
\left|d^{\prime}(t)\right| \leq C\left\{e^{-c_{-}^{\prime}\left(\frac{1}{2} s_{1} t+\left|d_{0}\right|\right)}+e^{-c_{+}\left(|s| t+x_{1}\right)}\right\} .
$$

This implies that $d^{\prime}(t) \in L^{1}(0, \infty)$. Further, we get the boundedness of $|d(t)|$

$$
|d(t)| \leq|d(0)|+\int_{0}^{t}\left|d^{\prime}(\tau)\right| d \tau \leq C
$$


Thus, based on $|d(t)| \leq C$, Eq. (3.4), (4.21), and Lemmas 2.1 and 2.3, we obtain (4.12).

Finally, $d_{\infty}$ can be calculated as showed by Matsumura and Mei in [26], and we can prove $d(t) \rightarrow d_{\infty}$ by the continuity as $t \rightarrow \infty$. We omit the details. The proof is completed.

Now, we are going to prove the basic energy estimate.

LEMMA 4.3. Under the assumptions of Theorem 4.1, it holds, for any solution $w(x, t)$ of (4.7) and (4.9) with $w \in X(0, T)$, that

$$
\begin{aligned}
& \left\|\left(w, \sqrt{a} w_{x}, w_{t}\right)(\cdot, t)\right\|^{2}+\int_{0}^{t}\left\|\left(\sqrt{U_{s_{1}}^{\prime}} w, \sqrt{a} w_{x}, w_{t}\right)(\cdot, \tau)\right\|^{2} d \tau \\
\leq & C\left(e^{-c_{+} x_{1} / 4}+e^{c_{-}^{\prime} d_{0} / 4}+\left\|\left(w_{0}, w_{0 x}, z_{0}\right)\right\|^{2}\right) .
\end{aligned}
$$

provided that $N(T)+\left|d_{0}\right|^{-1}+x_{1}^{-1} \ll 1$.

Proof. Let us denote

$$
L(w):=w_{t t}+w_{t}-a w_{x x}+f^{\prime}\left(U_{p}\right) w_{x},
$$

and consider the equality

$$
Q\left(w+2 w_{t}\right) \cdot L(w)=Q\left(w+2 w_{t}\right)\left(g_{1}+g_{2}\right) .
$$

The left-hand side of (4.22) can be reduced to

$$
\begin{aligned}
& Q\left(w+2 w_{t}\right) L(w) \\
= & \partial_{t}\left\{Q\left(w_{t}^{2}+w w_{t}+\frac{1}{2}\left(1-Q_{t} / Q\right) w^{2}\right)+a Q w_{x}^{2}\right\} \\
+ & Q\left\{w_{t}^{2}\left(1-Q_{t} / Q\right)+2\left(f^{\prime}\left(U_{p}\right)+a Q_{x} / Q\right) w w_{t}+a\left(1-Q_{t} / Q\right) w_{x}^{2}\right\} \\
& -\partial_{x}\left\{a Q w w_{x}-\frac{1}{2} a Q_{x} w^{2}-\frac{1}{2} Q f^{\prime}\left(U_{p}\right) w^{2}+2 a Q w_{t} w_{x}\right\} \\
+ & \frac{1}{2} w^{2}\left\{Q_{t t}-Q_{t}-a Q_{x x}-\left(Q f^{\prime}\left(U_{p}\right)\right)_{x}\right\} \\
= & \partial_{t}\left\{Q\left(w_{t}^{2}+w w_{t}+\frac{1}{2}\left(1+\left(s_{1}-d^{\prime}(t)\right) Q_{x} / Q\right) w^{2}\right)+a Q w_{x}^{2}\right\} \\
+ & Q\left\{w_{t}^{2}\left(1+\left(s_{1}-d^{\prime}(t)\right) Q_{x} / Q\right)+2\left(f^{\prime}\left(U_{p}\right)+a Q_{x} / Q\right) w_{x} w_{t}\right. \\
& \left.\quad+\left(1+\left(s_{1}-d^{\prime}(t)\right) Q_{x} / Q\right) a w_{x}^{2}\right\} \\
- & \partial_{x}\left\{a Q w w_{t}-\frac{1}{2} a Q_{x} w^{2}-\frac{1}{2} Q f^{\prime}\left(U_{p}\right) w^{2}+2 a Q w_{t} w_{x}\right. \\
& \left.\quad-\frac{1}{2} Q_{x} d^{\prime}(t)\left(2 s_{1}-d^{\prime}(t)\right) w^{2}\right\} \\
+ & \frac{1}{2} w^{2}\left(Q h_{1}\right)^{\prime \prime} U_{s_{1}}^{\prime} \\
+ & \left\{Q_{x} d^{\prime}(t)\left(2 s_{1}-d^{\prime}(t)\right) w w_{x}-\frac{1}{2} w^{2} \partial_{x}\left\{Q\left(f^{\prime}\left(U_{p}\right)-f^{\prime}\left(U_{s_{1}}\right)\right)+d^{\prime}(t) Q\right\}\right\}
\end{aligned}
$$




$$
\begin{aligned}
= & : \partial_{t}\left\{G_{1}\left(w, w_{t}\right)+G_{2}\left(w_{x}\right)\right\}+G_{3}\left(w_{t}, w_{x}\right)-\partial_{x} G_{4}\left(w, w_{t}, w_{x}\right) \\
& +G_{5}(w)+G_{6}\left(w, w_{x}\right) .
\end{aligned}
$$

As $a>0$ is big enough, it holds $\left|Q_{x} / Q\right|=O(1) \frac{u_{+}-u_{-}^{1}}{a-s_{1}^{2}} \ll 1$. With a similar argument in $[28,29,17]$, we gain

$$
\begin{aligned}
& \Delta_{1}=: 1-2\left[1+\left(s_{1}-d^{\prime}(t)\right) \frac{Q_{x}}{Q}\right]=-\left[1+2\left(s_{1}-d^{\prime}(t)\right) \frac{Q_{x}}{Q}\right] \leq-C<0, \\
& \Delta_{3}=: 4\left(f^{\prime}\left(U_{p}\right)+a \frac{Q_{x}}{Q}\right)^{2}-4 a\left(1+\left(s_{1}-d^{\prime}(t)\right) \frac{Q_{x}}{Q}\right)^{2} \leq-C<0
\end{aligned}
$$

provided that $\left|d_{0}\right|^{-1}+x_{1}^{-1} \ll 1$, where $\Delta_{1}$ and $\Delta_{3}$ are the discriminates of $G_{1}$ and $G_{3}$ respectively. Thus, we have

$$
\begin{aligned}
& G_{1}\left(w, w_{t}\right)+G_{2}\left(w_{x}\right) \geq C^{-1}\left(w^{2}+w_{t}^{2}+a w_{x}^{2}\right), \\
& G_{3}\left(w, w_{t}\right) \geq C^{-1}\left(w_{x}^{2}+w_{t}^{2}\right),
\end{aligned}
$$

By (2.12) and Lemma 2.3, we have

$$
G_{5}(w)=U_{s_{1}}^{\prime} w^{2} .
$$

Integrating (4.22) over $[0,+\infty) \times[0, t]$, using (4.26)-(4.28), and the zero-boundary conditions $\left.w\right|_{x=0}=\left.w\right|_{x=\infty}=\left.w_{t}\right|_{x=0}=\left.w_{t}\right|_{x=\infty}=0$ which implies $\left.G_{4}\right|_{x=0}=\left.G_{4}\right|_{x=\infty}$ $=0$, we obtain

$$
\begin{aligned}
& \left\|\left(w, \sqrt{a} w_{x}, w_{t}\right)(\cdot, t)\right\|^{2}+\int_{0}^{t}\left\|\left(\sqrt{U_{s_{1}}^{\prime}} w, \sqrt{a} w_{x}, w_{t}\right)(\cdot, \tau)\right\|^{2} d \tau \\
\leq & C\left(\left|\int_{0}^{t} \int_{0}^{+\infty} Q\left(w+2 w_{t}\right)\left(g_{1}+g_{2}\right) d x d \tau\right|+\int_{0}^{t} \int_{0}^{+\infty}\left|G_{6}\left(w, w_{x}\right)\right| d x d \tau\right) \\
& +C\left\|\left(w_{0}, w_{0 x}, z_{0}\right)\right\|^{2} .
\end{aligned}
$$

By Lemmas 2.1-2.3 and Lemma 4.2, the term $G_{6}\left(w, w_{x}\right)$ can be estimated as

$$
\left|G_{6}\left(w, w_{x}\right)\right| \leq C\left(N(T) w_{x}^{2}+\left|d^{\prime}(t) U_{s_{1}}^{\prime}\right|+\left|U_{s}-u_{+}\right|+\left|U_{s}^{\prime}\right|\right),
$$

which implies

$$
\begin{aligned}
& \int_{0}^{t} \int_{0}^{+\infty}\left|G_{6}\left(w, w_{x}\right)\right| d x d \tau \\
\leq & C \int_{0}^{t} \int_{0}^{+\infty}\left|d^{\prime}(\tau)\right|\left(\int_{0}^{-\eta_{1}} \exp \left\{c_{-}^{\prime}\left(x+\eta_{1}\right)\right\} d x+\int_{-\eta_{1}}^{+\infty} \exp \left\{-c_{+}^{\prime}\left(x+\eta_{1}\right)\right\}\right) d x d \tau \\
& +C \int_{0}^{t} \int_{0}^{+\infty} C e^{-c_{+}\left(x-s t+x_{1}\right)} d x d \tau+C N(T) \int_{0}^{t} \int_{0}^{+\infty} w_{x}^{2} d x d \tau
\end{aligned}
$$

$$
\leq C N(T) \int_{0}^{t} \int_{0}^{+\infty} w_{x}^{2} d x d \tau+C\left(e^{-c_{+} x_{1} / 4}+e^{c_{-}^{\prime} d_{0} / 4}\right)
$$

provided that $\left|d_{0}\right|^{-1}+x_{1}^{-1} \ll 1$. 
Due to the Taylor's formula, it follows from (4.8), that

$$
\begin{aligned}
\left|g_{2}(x, t)\right| \leq & \left|f\left(U_{p}+w_{x}\right)-f\left(U_{p}\right)-f^{\prime}\left(U_{p}\right) w_{x}\right|+\left|f\left(U_{p}\right)-f\left(U_{s_{1}}\right)\right| \\
& +\left|f\left(U_{s}\right)-f\left(u_{+}\right)\right| \\
\leq & C w_{x}^{2}+C e^{-c_{+}\left(x-s t+x_{1}\right)} .
\end{aligned}
$$

Thus, using (4.32) and Lemmas 2.1-2.4, we can estimate the first term in the righthand side of (4.29) on $g_{2}(x, t)$, via Cauchy inequality and Sobolev inequality, as follows

$$
\begin{aligned}
& C\left|\int_{0}^{t} \int_{0}^{+\infty}\left(w+2 w_{t}\right) g_{2} d x d \tau\right| \\
\leq & C N(T) \int_{0}^{t} \int_{0}^{+\infty}\left(w_{t}^{2}+w_{x}^{2}\right) d x d \tau+C e^{-c_{+} x_{1} / 2} .
\end{aligned}
$$

Similarly, noticing

$$
\begin{aligned}
& C \int_{0}^{t} \int_{0}^{+\infty}\left|d^{\prime}(\tau)\right|\left(\left|U_{s_{1}}^{\prime}\left(x+\eta_{1}\right)\right|+\left|U_{s_{1}}\left(x+\eta_{1}\right)-u_{+}\right|\right) d x d \tau \\
\leq & C \int_{0}^{t}\left|d^{\prime}(\tau)\right|\left(\int_{0}^{-\eta_{1}}\left(\exp \left\{c_{-}^{\prime}\left(x+\eta_{1}\right)\right\}+1\right) d x+\int_{-\eta_{1}}^{+\infty} \exp \left\{-c_{+}^{\prime}\left(x+\eta_{1}\right)\right\}\right) d x d \tau \\
\leq & C\left(e^{-c_{+} x_{1} / 4}+e^{c_{-}^{\prime} d_{0} / 4}\right)
\end{aligned}
$$

we have

$$
\begin{aligned}
& C\left|\int_{0}^{t} \int_{0}^{+\infty} Q\left(w+2 w_{t}\right) g_{1} d x d \tau\right| \\
\leq & \frac{1}{4} \int_{0}^{t} \int_{0}^{+\infty} w_{t}^{2} d x d \tau \\
& +C \int_{0}^{t} \int_{0}^{+\infty}\left|d^{\prime}(\tau)\right|\left(\left|U_{s_{1}}^{\prime}\left(x+\eta_{1}\right)\right|+\left|V_{s_{1}}^{\prime}\left(x+\eta_{1}\right)\right|+\left|U_{s_{1}}\left(x+\eta_{1}\right)-u_{+}\right|\right) \mid d x d \tau
\end{aligned}
$$

$$
\leq \frac{1}{4} \int_{0}^{t} \int_{0}^{+\infty} w_{t}^{2} d x d \tau+C\left(e^{-c_{+} x_{1} / 4}+e^{c_{-}^{\prime} d_{0} / 4}\right) .
$$

With the help of (4.31), (4.33) and (4.34), we obtain Lemma 4.3 from (4.29), provided $N(T)+\left|d_{0}\right|^{-1}+x_{1}^{-1} \ll 1$.

Now, we turn to the higher order energy estimate. We have

LEMMA 4.4. Under the assumptions of Theorem 4.1, it holds, for any solution $w(x, t)$ of the IBVP (4.7) and (4.9) with $w \in X(0, T)$, that

$$
\begin{aligned}
& \left\|\left(w_{t}, \sqrt{a} w_{x x}, w_{x t}\right)(\cdot, t)\right\|^{2}+\int_{0}^{t}\left\|\left(w_{t}, \sqrt{a} w_{x x}, w_{x t}\right)(\cdot, \tau)\right\|^{2} d \tau \\
\leq & C\left(e^{-c_{+} x_{1} / 4}+e^{c_{-}^{\prime} d_{0} / 4}+\left\|\left(w_{0}, w_{0 x}, z_{0}\right)\right\|_{1}^{2}\right) .
\end{aligned}
$$

provided that $N(T)+\left|d_{0}\right|^{-1}+x_{1}^{-1} \ll 1$. 
Proof. Consider the equality

$$
Q\left(w_{t}+2 w_{t t}\right) \partial_{t} L(w)=Q\left(w_{t}+2 w_{t t}\right)\left(g_{1 t}+g_{2 t}\right) .
$$

Integrating $(4.35)$ over $[0,+\infty) \times[0, t]$, using the similar argument as used in Lemma 4.3 and the zero-boundary condition $\left.w\right|_{x=0}=\left.w\right|_{x=\infty}=\left.w_{t}\right|_{x=0}=\left.w_{t}\right|_{x=\infty}=0$, we obtain

$$
\begin{aligned}
& \left\|\left(w_{t}, \sqrt{a} w_{x t}, w_{t t}\right)(\cdot, t)\right\|^{2}+\int_{0}^{t}\left\|\left(w_{t}, \sqrt{a} w_{x t}, w_{t t}\right)(\cdot, \tau)\right\|^{2} d \tau \\
\leq & C\left\|\left(w_{0}, w_{0 x}, z_{0}\right)\right\|_{1}^{2}+C\left|\int_{0}^{t} \int_{0}^{+\infty}\left(w_{t}+2 w_{t t}\right)\left(g_{1 t}+g_{2 t}\right) d x d \tau\right| \\
& +C\left|\int_{0}^{t} \int_{0}^{+\infty}\left(w_{t}+2 w_{t t}\right) f^{\prime \prime}\left(U_{p}\right) \partial_{t} U_{p} w_{x} d x d \tau\right| .
\end{aligned}
$$

The last term in the right-hand side of (4.36) can be estimated as

$$
\begin{aligned}
& C\left|\int_{0}^{t} \int_{0}^{+\infty}\left(w_{t}+2 w_{t t}\right) f^{\prime \prime}\left(U_{p}\right) \partial_{t} U_{p} w_{x} d x d \tau\right| \\
\leq & C \int_{0}^{t} \int_{0}^{+\infty}\left(w_{t}^{2}+w_{x}^{2}\right) d x d \tau+\frac{1}{2} \int_{0}^{t} \int_{0}^{+\infty} w_{t t}^{2} d x d \tau \\
\leq & C\left(e^{-c_{+} x_{1} / 4}+e^{c_{-}^{\prime} d_{0} / 4}+\left\|\left(w_{0}, w_{0 x}, z_{0}\right)\right\|^{2}\right)+\frac{1}{2} \int_{0}^{t} \int_{0}^{+\infty} w_{t t}^{2} d x d \tau .
\end{aligned}
$$

After a tedious computation on $\left|g_{i t}(x, t)\right|,(i=1,2)$, to get

$$
\begin{aligned}
\left|g_{1 t}(x, t)\right| \leq C( & \left(\left|d^{\prime}(t)\right|+\left|d^{\prime \prime}(t)\right|\right)\left|U_{s_{1}}^{\prime}\left(x+\eta_{1}\right)\right| \\
& \left.+\left(\left|d^{\prime \prime}(t)\right|+\left|d^{\prime \prime \prime}(t)\right|\right)\left|U_{s_{1}}^{\prime}\left(x+\eta_{1}\right)-u_{+}\right|\right) \\
\left|g_{2 t}(x, t)\right| \leq C( & \left(\left|U_{s_{1}}^{\prime}\left(x+\eta_{1}\right)\right|+\left|U_{s}^{\prime}\left(x+\eta_{2}\right)\right|\right)\left|U_{s}^{\prime}\left(x+\eta_{2}\right)-u_{+}\right| \\
& +\left|U_{s}^{\prime}\left(x+\eta_{2}\right)\right|+\left|w_{x} w_{x t}\right| \\
& \left.+\left(\left|w_{x t}\right|+\left|U_{s_{1}}^{\prime}\left(x+\eta_{1}\right)\right|+\left|U_{s}^{\prime}\left(x+\eta_{2}\right)\right|\right) w_{x}^{2}\right)
\end{aligned}
$$

we can estimate the second term in the right-hand side of (4.36), with the help of Cauchy inequality and Sobolev embedding theorem, as

$$
\begin{aligned}
& C\left|\int_{0}^{t} \int_{0}^{+\infty} Q\left(w_{t}+2 w_{t t}\right)\left(g_{1 t}+g_{2 t}\right) d x d \tau\right| \\
\leq & \int_{0}^{t} \int_{0}^{+\infty}\left(w_{t}^{2}+w_{x}^{2}\right) d x d \tau+\frac{1}{4} \int_{0}^{t} \int_{0}^{+\infty}\left(w_{t t}^{2}+w_{x t}^{2}\right) d x d \tau \\
& +C\left(e^{-c_{+} x_{1} / 4}+e^{c_{-}^{\prime} d_{0} / 4}+\left\|\left(w_{0}, w_{0 x}, z_{0}\right)\right\|^{2}\right) \\
\leq & C\left(e^{-c_{+} x_{1} / 4}+e^{c_{-}^{\prime} d_{0} / 4}+\left\|\left(w_{0}, w_{0 x}, z_{0}\right)\right\|^{2}\right)+\frac{1}{4} \int_{0}^{t} \int_{0}^{+\infty}\left(w_{t t}^{2}+w_{x t}^{2}\right) d x d \tau .
\end{aligned}
$$


Substituting (4.37) and (4.38) into (4.36), we obtain

$$
\begin{aligned}
& \left\|\left(w_{t}, \sqrt{a} w_{x t}, w_{t t}\right)(\cdot, t)\right\|^{2}+\int_{0}^{t}\left\|\left(w_{t}, \sqrt{a} w_{x t}, w_{t t}\right)(\cdot, \tau)\right\|^{2} d \tau \\
\leq & C\left(e^{-c_{+} x_{1} / 4}+e^{c_{-}^{\prime} d_{0} / 4}+\left\|\left(w_{0}, w_{0 x}, z_{0}\right)\right\|_{1}^{2}\right),
\end{aligned}
$$

which yields Lemma 2.4 with the help of Lemma 4.3 and the fact

$$
a w_{x x}=w_{t t}+w_{t}+f^{\prime}\left(U_{p}\right) w_{x}-\left(g_{1}+g_{2}\right) .
$$

Then, Theorem 4.1 can be proved in terms of Lemmas 4.3-4.4, the local existence, and the standard continuity argument.

ACKNOWLEDGEMENT. The authors thank referees for the helpful suggestions and comments on the orginal manuscript.

\section{REFERENCES}

[1] T. Chang And L. Hsiao, Riemann Problem and Interaction of Waves in Gas Dynamics, Pitman Monographs and Surveys in Pure and Applied Mathematics 11, Longman Scientific and Technical Essex, 1989.

[2] G.-Q. Cheng, C. D. Levermore, and T. P. LiU, Hyperbolic conservation laws with stiff relaxation terms and entropy, Comm. Pure Appl. Math., 47 (1994), pp. 787-830.

[3] G.-Q. CHEN AND T.-P. LIU, Zero relaxation and dissipation limits for hyperbolic conservation laws, Comm. Pure Appl. Math., 46 (1993), pp. 755-781.

[4] I.-L. ChenN, Long-time effect of relaxation for hyperbolic conservation laws, Comm. Math. Phys., 172 (1995), pp. 39-55.

[5] J. F. Clarke, Gas dynamics with relaxation effects, Rep. Prog. Phys., 41 (1978), pp. 807-863.

[6] L. HSIAO, H. LI, AND M. MEI, Convergence rates to superposition of two travelling waves of the solutions to a relaxation hyperbolic system with boundary effects, Preprint.

[7] L. Hsiao AND R. H. PAN, Nonlinear stability of two-mode shock profiles for a rate-type viscoelastic system with relaxation, Chin. Annl. Math., to appear.

[8] S. JiN, A convex entropy for a hyperbolic system with stiff relaxation, J. Differential Equations, 127 (1996), pp. 97-109.

[9] S. Jin AND C. D. LeVERMORE, Numerical schemes for systems of conservation laws with stiff relaxation terms, J. Comput. Phys., 126 (1996), pp. 449-467.

[10] S. Jin AND H. LiU, Diffusion limit of a hyperbolic system with relaxation, Methods Appl. Anal., 5 (1998), pp. 317-334.

[11] S. JIN AND Z. P. XIN, The relaxation schemes for systems of conservation laws in arbitrary space dimensions, Comm. Pure Appl. Math., 48 (1995), pp. 23.5-277.

[12] S. KaWAshima And A. Matsumura, Asymptotic stability to travelling wave solutions of systems for one-dimensional gas motion, Comm. Math. Phys., 101 (1985), pp. 97-127.

[13] S. KaWAShima AND A. Matsumura, Stability of shock profiles in viscoelasticity with nonconvex constitutive relations, Comm. Pure Appl. Math., 47 (1994), pp. 1547-1569.

[14] H. LI, Global existence and large time behavior of solution to reacting flow model with boundary effect, Acta Math. Sci., to appear.

[15] D. Q. Li AND W. C. YU, Boundary Value Problems for Quasilinear Hyperbolic Systems, Duke University Mathematics Series V, 1985.

[16] H. LIU, J. WANG, AND T. YANG, Stability in a relaxation model with a nonvonvex flux, SIAM J. Math., 29 (1998), pp. 18-29.

[17] H. LIU, C. W. WoO, AND T. YANG, Decay rate for travelling waves of a relaxation model, J. Differential Equations, 134 (1997), pp. 343-367.

[18] J. G. LIU AND Z. XIN, Kinetic and viscous boundary layers for broadwell equations, Trans. Theory Statist. Phys., 25 (1996), pp. 447-461.

[19] J. G. LIU AND Z. P. XIN, Boundary layer behavior in the fluid-dynamic limit for a nonlinear model Boltzmann equation, Arch. Rational Mech. Anal., 136 (1996), pp. 51-101.

[20] T.-P. LIU, Hyperbolic conservation laws with relaxation, Comm. Math. Phys., 108 (1987), pp. 153-175. 
[21] T.-P. LIU AND K. NishiHARA, Asymptotic behavior for scalar viscous conservation laws with boundary effect, J. Differential Equations, 133 (1997), pp. 296-320.

[22] T.-P. LiU AND S.-H. YU, Propagation of a stationary shock layer in the presence of a boundary, Arch. Rational Mech. Anal., 139 (1997), pp. 296-320.

[23] T. LUO AND Z. XIN, Asymptotic stability of planar shock profiles for the relaxation approximations of conservation laws in several dimensions, J. Differential Equations, 139 (1997), pp. 365-408.

[24] P. MARCATI AND B. RUBINo, Hyperbolic to parabolic theory for quasilinear first order systems, to appear.

[25] C. MASCIA AND R. NATALINI, $L^{1}$ nonlinear stability of travelling waves for a hyperbolic system with relaxation, J. Differential Equations, 132 (1996), pp. 275-292.

[26] A. Matsumura AND M. MEI, Convergence to travelling fronts of solutions of the p-system with viscosity in the presence of a boundary, Arch. Rational Mech. Anal., 146 (1999), pp. 1-22.

[27] A. Matsumura AND K. Nishihara, Asymptotic stability of travelling waves for scalar viscous conservation laws with nonconvex nonlinearity, Comm. Math. Phys., 165 (1994), pp. 83-96.

[28] M. Mei AND B. Rubino, Convergence to traveling waves with decay rates for solution of the initial boundary value problem to a nonconvex relaxation model, J. Differential Equations, 1999 , in press.

[29] M. MEI AND T. YANG, Convergence rates to travelling waves for a nonconvex relaxation model, Proc. Roy. Soc. Edinburgh Sect. A, 128 (1998), pp. 1053-1068.

[30] R. NATALINI, Convergence to equilibrium for the relaxation approximations of conservation laws, Comm. Pure Appl. Math., 49 (1996), pp. 795-823.

[31] S. NishiBATA, The initial boundary value problems for hyperbolic conservation laws with relaxation, J. Differential Equations, 130 (1996), pp. 100-126.

[32] M. NishikAWA, Convergence rate to travelling waves for viscous conservation laws, Funkical. Ekvac., 41 (1998), pp. 107-132.

[33] S. Nishibata AND S.-H. Yu, The asymptotic behavior of the hyperbolic conservation laws with relaxation on the quarter-plane, SIAM J. Math. Anal., 28 (1997), pp. 304-321.

[34] H. Schlichting, Boundary-Layer Theory, 7th edition, McGraw-Hill, 1979.

[35] W. C. WANG AND Z. P. XIN, Asymptotic limit of initial boundary value problems for conservation laws with relaxation extensions, Comm. Pure Appl. Math., 51 (1998), pp. 505-535.

[36] G. Whiтнам, Linear and nonlinear waves, Wiley-Interscience, 1974.

[37] Z. XIN, Viscous boundary layer and their stability, J. Partial Differential Equation, 11 (1998), pp. 97-124.

[38] Z. A. YAO AND C. J. ZHU, $L^{p}$-convergence rate for $p$-system with relaxation, to appear.

[39] P. Zingano, Nonlinear stability with decay rate for travelling wave solutions of a hyperbolic system with relaxation, J. Differential Equations, 130 (1996), pp. 36-58. 
